\title{
Some vector inequalities for two operators in Hilbert spaces with applications
}

\author{
Sever S. Dragomir \\ Mathematics, School of Engineering \& Science \\ Victoria University, Australia \\ School of Computational \& Applied Mathematics, \\ University of the Witwatersrand, South Africa \\ email: sever.dragomir@vu.edu.au
}

\begin{abstract}
In this paper we establish some vector inequalities for two operators related to Schwarz and Buzano results. We show amongst others that in a Hilbert space $\mathrm{H}$ we have the inequality

$$
\begin{aligned}
\frac{1}{2}\left[\left\langle\frac{|A|^{2}+|B|^{2}}{2} x, x\right\rangle^{1 / 2}\right. & \left.\left\langle\frac{|A|^{2}+|B|^{2}}{2} y, y\right\rangle^{1 / 2}+\left|\left\langle\frac{|A|^{2}+|B|^{2}}{2} x, y\right\rangle\right|\right] \\
& \geq\left|\left\langle\operatorname{Re}\left(B^{*} A\right) x, y\right\rangle\right|
\end{aligned}
$$
\end{abstract}

for $A, B$ two bounded linear operators on $H$ such that $\operatorname{Re}\left(B^{*} A\right)$ is a nonnegative operator and any vectors $x, y \in \mathrm{H}$.

Applications for norm and numerical radius inequalities are given as well.

\section{Introduction}

Let $(H,\langle\cdot, \cdot\rangle)$ be an inner product space over the real or complex numbers field $\mathbb{K}$. The following inequality is well known in literature as the Schwarz inequality

$$
\|x\|\|y\| \geq|\langle x, y\rangle| \text { for any } x, y \in H .
$$

2010 Mathematics Subject Classification: 46C05, 26D15, 26D1

Key words and phrases: inner product spaces, Schwarz's inequality, Buzano's inequality, projection, selfadjoint operators, unitary operators, operator norm, numerical radius 
The equality case holds in (1) if and only if there exists a constant $\lambda \in \mathbb{K}$ such that $x=\lambda y$.

In 1985 the author [5] (see also [24]) established the following refinement of (1):

$$
\|x\|\|y\| \geq|\langle x, y\rangle-\langle x, e\rangle\langle e, y\rangle|+|\langle x, e\rangle\langle e, y\rangle| \geq|\langle x, y\rangle|
$$

for any $x, y, e \in H$ with $\|e\|=1$.

Using the triangle inequality for modulus we have

$$
|\langle x, y\rangle-\langle x, e\rangle\langle e, y\rangle| \geq|\langle x, e\rangle\langle e, y\rangle|-|\langle x, y\rangle|
$$

and by (2) we get

$$
\begin{aligned}
\|x\|\|y\| & \geq|\langle x, y\rangle-\langle x, e\rangle\langle e, y\rangle|+|\langle x, e\rangle\langle e, y\rangle| \\
& \geq 2|\langle x, e\rangle\langle e, y\rangle|-|\langle x, y\rangle|
\end{aligned}
$$

which implies the Buzano inequality [2]

$$
\frac{1}{2}[\|x\|\|y\|+|\langle x, y\rangle|] \geq|\langle x, e\rangle\langle e, y\rangle|
$$

that holds for any $x, y, e \in H$ with $\|e\|=1$.

A family $\left\{e_{j}\right\}_{j \in J}$ of vectors in $\mathrm{H}$ is called orthonormal if

$$
e_{j} \perp e_{k} \text { for any } j, k \in J \text { with } j \neq k \text { and }\left\|e_{j}\right\|=1 \text { for any } j, k \in J .
$$

If the linear span of the family $\left\{\boldsymbol{e}_{\mathrm{j}}\right\}_{\mathrm{j} \in \mathrm{J}}$ is dense in $\mathrm{H}$, then we call it an orthonormal basis in $\mathrm{H}$.

It is well known that for any orthonormal family $\left\{\boldsymbol{e}_{j}\right\}_{j \in \mathrm{J}}$ we have Bessel's inequality

$$
\sum_{j \in J}\left|\left\langle x, e_{j}\right\rangle\right|^{2} \leq\|x\|^{2} \text { for any } x \in H .
$$

This becomes Parseval's identity

$$
\sum_{j \in J}\left|\left\langle x, e_{j}\right\rangle\right|^{2}=\|x\|^{2} \text { for any } x \in H
$$

when $\left\{\boldsymbol{e}_{\mathrm{j}}\right\}_{\mathfrak{j} \in \mathrm{J}}$ an othonormal basis in $\mathrm{H}$.

For an othonormal family $\mathcal{E}=\left\{\boldsymbol{e}_{\mathrm{j}}\right\}_{j \in \mathrm{J}}$ we define the operator $\mathrm{P}_{\mathcal{E}}: \mathrm{H} \rightarrow \mathrm{H}$ by

$$
P_{\mathcal{E}} x:=\sum_{j \in J}\left\langle x, e_{j}\right\rangle e_{j}, x \in H
$$


We know that $\mathrm{P}_{\mathcal{E}}$ is an orthogonal projection and

$$
\left\langle\mathrm{P}_{\mathcal{E}} \chi, y\right\rangle=\sum_{j \in J}\left\langle x, e_{j}\right\rangle\left\langle e_{j}, y\right\rangle, x, y \in H \text { and }\left\langle P_{\mathcal{E}} x, x\right\rangle=\sum_{j \in J}\left|\left\langle x, e_{j}\right\rangle\right|^{2}, x \in H .
$$

The particular case when the family reduces to one vector, namely $\mathcal{E}=\{e\}$, $\|e\|=1$, is of interest since in this case $P_{e} x:=\langle x, e\rangle e, x \in H$,

$$
\left\langle P_{e} x, y\right\rangle=\langle x, e\rangle\langle e, y\rangle, x, y \in H
$$

and Buzano's inequality can be written as

$$
\frac{1}{2}[\|x\|\|y\|+|\langle x, y\rangle|] \geq\left|\left\langle P_{e} x, y\right\rangle\right|
$$

that holds for any $x, y, e \in \mathrm{H}$ with $\|e\|=1$.

In an effort to generalize the inequality (6) for general projection, in [21] we obtained the following result

$$
\frac{1}{2}[\|x\|\|y\|+|\langle x, y\rangle|] \geq|\langle P x, y\rangle|
$$

for any $x, y \in H$ and $P: H \rightarrow H$ a projection on $H$.

In particular, we then have the inequality

$$
\frac{1}{2}[\|x\|\|y\|+|\langle x, y\rangle|] \geq\left|\left\langle\sum_{j \in J}\left\langle x, e_{j}\right\rangle\left\langle e_{j}, y\right\rangle\right\rangle\right|
$$

for any orthonormal family $\left\{\boldsymbol{e}_{\mathrm{j}}\right\}_{\mathrm{j} \in \mathrm{J}}$ and any $x, y \in \mathrm{H}$.

Motivated by the above results we establish in this paper some vector inequalities for two operators $A, B$ for which the operator $\operatorname{Re}\left(B^{*} A\right)$ is nonnegative in the operator order that are related to the inequality (6). Applications for norm and numerical radius inequalities are provided as well.

For other Schwarz and Buzano related inequalities in inner product spaces, see [1]-[4], [5]-[14], [22]-[26], [30]-[39], and the monographs [16], [17] and [18].

\section{Vector inequalities for two operators}

For a bounded linear operator $\mathrm{T}$ we use the concepts of absolute value and real part of $\mathrm{T}$ defined as

$$
|\mathrm{T}|=\left(\mathrm{T}^{*} \mathrm{~T}\right)^{1 / 2} \text { and } \operatorname{Re}(\mathrm{T})=\frac{\mathrm{T}+\mathrm{T}^{*}}{2} .
$$

We have the following vector inequality: 
Theorem 1 Let A, B two bounded linear operators on $\mathrm{H}$ such that $\operatorname{Re}\left(\mathrm{B}^{*} \mathrm{~A}\right)$ is a nonnegative operator. Then for any $x, y \in \mathrm{H}$ we have the inequality

$$
\begin{aligned}
& \left\langle\frac{|A|^{2}+|B|^{2}}{2} x, x\right\rangle^{1 / 2}\left\langle\frac{|A|^{2}+|B|^{2}}{2} y, y\right\rangle^{1 / 2} \\
& \geq\left\langle\operatorname{Re}\left(B^{*} A\right) x, x\right\rangle^{1 / 2}\left\langle\operatorname{Re}\left(B^{*} A\right) y, y\right\rangle^{1 / 2} \\
& +\left|\left\langle\frac{|A|^{2}+|B|^{2}}{2} x, y\right\rangle-\left\langle\operatorname{Re}\left(B^{*} A\right) x, y\right\rangle\right| .
\end{aligned}
$$

Proof. Using Schwarz inequality we have

$$
\|A x-B x\|^{2}\|A y-B y\|^{2} \geq|\langle A x-B x, A y-B y\rangle|^{2}
$$

for any $x, y \in H$.

Observe that

$$
\begin{aligned}
\|A x-B x\|^{2} & =\langle A x, A x\rangle-\langle A x, B x\rangle-\langle B x, A x\rangle+\langle B x, B x\rangle \\
& =\left\langle A^{*} A x, x\right\rangle-\left\langle B^{*} A x, x\right\rangle-\left\langle A^{*} B x, x\right\rangle+\left\langle B^{*} B x, x\right\rangle \\
& =\left\langle|A|^{2} x, x\right\rangle+\left\langle|B|^{2} x, x\right\rangle-\left\langle\left(B^{*} A+A^{*} B\right) x, x\right\rangle \\
& =2\left[\left\langle\frac{|A|^{2}+|B|^{2}}{2} x, x\right\rangle-\left\langle\operatorname{Re}\left(B^{*} A\right) x, x\right\rangle\right] \geq 0
\end{aligned}
$$

and, similarly,

$$
\|A y-B y\|^{2}=2\left[\left\langle\frac{|A|^{2}+|B|^{2}}{2} y, y\right\rangle-\left\langle\operatorname{Re}\left(B^{*} A\right) y, y\right\rangle\right] \geq 0
$$

for any $x, y \in H$.

We also have

$$
\langle A x-B x, A y-B y\rangle=2\left[\left\langle\frac{|A|^{2}+|B|^{2}}{2} x, y\right\rangle-\left\langle\operatorname{Re}\left(B^{*} A\right) x, y\right\rangle\right]
$$

for any $x, y \in H$.

Using the inequality (11) and the equalities (12)-(14) we get

$$
\begin{aligned}
& {\left[\left\langle\frac{|A|^{2}+|B|^{2}}{2} x, x\right\rangle-\left\langle\operatorname{Re}\left(B^{*} A\right) x, x\right\rangle\right]} \\
& \times\left[\left\langle\frac{|A|^{2}+|B|^{2}}{2} y, y\right\rangle-\left\langle\operatorname{Re}\left(B^{*} A\right) y, y\right\rangle\right] \\
& \geq\left|\left\langle\frac{|A|^{2}+|B|^{2}}{2} x, y\right\rangle-\left\langle\operatorname{Re}\left(B^{*} A\right) x, y\right\rangle\right|^{2}
\end{aligned}
$$


for any $x, y \in H$.

Since $\operatorname{Re}\left(B^{*} A\right) \geq 0$, then we have

$$
\left\langle\frac{|A|^{2}+|B|^{2}}{2} x, x\right\rangle \geq\left\langle\operatorname{Re}\left(B^{*} A\right) x, x\right\rangle \geq 0
$$

and

$$
\left\langle\frac{|A|^{2}+|B|^{2}}{2} y, y\right\rangle \geq\left\langle\operatorname{Re}\left(B^{*} A\right) y, y\right\rangle \geq 0
$$

for any $x, y \in H$.

Using the elementary inequality that holds for any real numbers $a, b, c, d$

$$
(a c-b d)^{2} \geq\left(a^{2}-b^{2}\right)\left(c^{2}-d^{2}\right),
$$

we have

$$
\begin{aligned}
& \left(\left\langle\frac{|A|^{2}+|B|^{2}}{2} x, x\right\rangle^{1 / 2}\left\langle\frac{|A|^{2}+|B|^{2}}{2} y, y\right\rangle^{1 / 2}\right. \\
& \left.-\left\langle\operatorname{Re}\left(B^{*} A\right) x, x\right\rangle^{1 / 2}\left\langle\operatorname{Re}\left(B^{*} A\right) y, y\right\rangle^{1 / 2}\right)^{2} \\
& \geq\left[\left\langle\frac{|A|^{2}+|B|^{2}}{2} x, x\right\rangle-\left\langle\operatorname{Re}\left(B^{*} A\right) x, x\right\rangle\right] \\
& \times\left[\left\langle\frac{|A|^{2}+|B|^{2}}{2} y, y\right\rangle-\left\langle\operatorname{Re}\left(B^{*} A\right) y, y\right\rangle\right]
\end{aligned}
$$

for any $x, y \in H$.

Making use of (15) and (16) we get

$$
\begin{aligned}
& \left(\left\langle\frac{|A|^{2}+|B|^{2}}{2} x, x\right\rangle^{1 / 2}\left\langle\frac{|A|^{2}+|B|^{2}}{2} y, y\right\rangle^{1 / 2}\right. \\
& -\left\langle\operatorname{Re}\left(B^{*} A x, x\right\rangle^{1 / 2}\left\langle\operatorname{Re}\left(B^{*} A\right) y, y\right\rangle^{1 / 2}\right)^{2} \\
& \geq\left|\left\langle\frac{|A|^{2}+|B|^{2}}{2} x, y\right\rangle-\left\langle\operatorname{Re}\left(B^{*} A\right) x, y\right\rangle\right|^{2}
\end{aligned}
$$

for any $x, y \in \mathrm{H}$.

Since

$$
\left\langle\frac{|A|^{2}+|B|^{2}}{2} x, x\right\rangle^{1 / 2}\left\langle\frac{|A|^{2}+|B|^{2}}{2} y, y\right\rangle^{1 / 2} \geq\left\langle\operatorname{Re}\left(B^{*} A\right) x, x\right\rangle^{1 / 2}\left\langle\operatorname{Re}\left(B^{*} A\right) y, y\right\rangle^{1 / 2}
$$

for any $x, y \in H$, then by taking the square root in (17) we get the desired result from (10). 
Corollary 1 With the assumptions in Theorem 1 we have

$$
\begin{aligned}
& \left\langle\frac{|A|^{2}+|B|^{2}}{2} x, x\right\rangle^{1 / 2}\left\langle\frac{|A|^{2}+|B|^{2}}{2} y, y\right\rangle^{1 / 2}-\left|\left\langle\frac{|A|^{2}+|B|^{2}}{2} x, y\right\rangle\right| \\
& \geq\left\langle\operatorname{Re}\left(B^{*} A\right) x, x\right\rangle^{1 / 2}\left\langle\operatorname{Re}\left(B^{*} A\right) y, y\right\rangle^{1 / 2}-\left|\left\langle\operatorname{Re}\left(B^{*} A\right) x, y\right\rangle\right| \geq 0
\end{aligned}
$$

and

$$
\begin{aligned}
& \left\langle\frac{|A|^{2}+|B|^{2}}{2} x, x\right\rangle^{1 / 2}\left\langle\frac{|A|^{2}+|B|^{2}}{2} y, y\right\rangle^{1 / 2}+\left|\left\langle\frac{|A|^{2}+|B|^{2}}{2} x, y\right\rangle\right| \\
& \geq\left\langle\operatorname{Re}\left(B^{*} A\right) x, x\right\rangle^{1 / 2}\left\langle\operatorname{Re}\left(B^{*} A\right) y, y\right\rangle^{1 / 2}+\left|\left\langle\operatorname{Re}\left(B^{*} A\right) x, y\right\rangle\right|
\end{aligned}
$$

for any $\mathrm{x}, \mathrm{y} \in \mathrm{H}$.

Proof. From the triangle inequality we have

$$
\left|\left\langle\frac{|A|^{2}+|B|^{2}}{2} x, y\right\rangle-\left\langle\operatorname{Re}\left(B^{*} A\right) x, y\right\rangle\right| \geq\left|\left\langle\frac{|A|^{2}+|B|^{2}}{2} x, y\right\rangle\right|-\left|\left\langle\operatorname{Re}\left(B^{*} A\right) x, y\right\rangle\right|
$$

and

$$
\left|\left\langle\frac{|A|^{2}+|B|^{2}}{2} x, y\right\rangle-\left\langle\operatorname{Re}\left(B^{*} A\right) x, y\right\rangle\right| \geq\left|\left\langle\operatorname{Re}\left(B^{*} A\right) x, y\right\rangle\right|-\left|\left\langle\frac{|A|^{2}+|B|^{2}}{2} x, y\right\rangle\right|
$$

for any $x, y \in H$, which together with (10) produce the inequalities (18) and (19).

Remark 1 With the assumptions in Theorem 1 we have

$$
\begin{aligned}
& \frac{1}{2}\left[\left\langle\frac{|A|^{2}+|B|^{2}}{2} x, x\right\rangle^{1 / 2}\left\langle\frac{|A|^{2}+|B|^{2}}{2} y, y\right\rangle^{1 / 2}\right. \\
& \left.+\left|\left\langle\frac{|A|^{2}+|B|^{2}}{2} x, y\right\rangle\right|\right] \geq\left|\left\langle\operatorname{Re}\left(B^{*} A\right) x, y\right\rangle\right|
\end{aligned}
$$

for any $\mathrm{x}, \mathrm{y} \in \mathrm{H}$.

If we assume that $A$ is a bounded linear operator such that $\operatorname{Re}\left(A^{2}\right) \geq 0$, then by taking $B=A^{*}$ above, we have the inequalities

$$
\begin{aligned}
& \left\langle\frac{|A|^{2}+\left|A^{*}\right|^{2}}{2} x, x\right\rangle^{1 / 2}\left\langle\frac{|A|^{2}+\left|A^{*}\right|^{2}}{2} y, y\right\rangle^{1 / 2} \\
& \geq\left\langle\operatorname{Re}\left(A^{2}\right) x, x\right\rangle^{1 / 2}\left\langle\operatorname{Re}\left(A^{2}\right) y, y\right\rangle^{1 / 2} \\
& +\left|\left\langle\frac{|A|^{2}+\left|A^{*}\right|^{2}}{2} x, y\right\rangle-\left\langle\operatorname{Re}\left(A^{2}\right) x, y\right\rangle\right|
\end{aligned}
$$




$$
\begin{aligned}
& \left\langle\frac{|A|^{2}+\left|A^{*}\right|^{2}}{2} x, x\right\rangle^{1 / 2}\left\langle\frac{|A|^{2}+\left|A^{*}\right|^{2}}{2} y, y\right\rangle^{1 / 2}-\left|\left\langle\frac{|A|^{2}+\left|A^{*}\right|^{2}}{2} x, y\right\rangle\right| \\
& \geq\left\langle\operatorname{Re}\left(A^{2}\right) x, x\right\rangle^{1 / 2}\left\langle\operatorname{Re}\left(A^{2}\right) y, y\right\rangle^{1 / 2}-\left|\left\langle\operatorname{Re}\left(A^{2}\right) x, y\right\rangle\right| \geq 0 \\
& \left\langle\frac{|A|^{2}+\left|A^{*}\right|^{2}}{2} x, x\right\rangle^{1 / 2}\left\langle\frac{|A|^{2}+\left|A^{*}\right|^{2}}{2} y, y\right\rangle^{1 / 2}+\left|\left\langle\frac{|A|^{2}+|B|^{2}}{2} x, y\right\rangle\right| \\
& \geq\left\langle\operatorname{Re}\left(A^{2}\right) x, x\right\rangle^{1 / 2}\left\langle\operatorname{Re}\left(A^{2}\right) y, y\right\rangle^{1 / 2}+\left|\left\langle\operatorname{Re}\left(A^{2}\right) x, y\right\rangle\right|
\end{aligned}
$$

and

$$
\begin{aligned}
& \frac{1}{2}\left[\left\langle\frac{|A|^{2}+\left|A^{*}\right|^{2}}{2} x, x\right\rangle^{1 / 2}\left\langle\frac{|A|^{2}+\left|A^{*}\right|^{2}}{2} y, y\right\rangle^{1 / 2}\right. \\
& \left.+\left|\left\langle\frac{|A|^{2}+\left|A^{*}\right|^{2}}{2} x, y\right\rangle\right|\right] \geq\left|\left\langle\operatorname{Re}\left(A^{2}\right) x, y\right\rangle\right|
\end{aligned}
$$

for any $x, y \in H$.

Assume that $A$ is invertible, then by selecting $B=\left(A^{-1}\right)^{*}$ above and taking into account that

$$
|B|^{2}=B^{*} B=A^{-1}\left(A^{-1}\right)^{*}=A^{-1}\left(A^{*}\right)^{-1}=\left(A^{*} A\right)^{-1}=|A|^{-2}
$$

then from the above we get the inequalities

$$
\begin{aligned}
& \left\langle\frac{|A|^{2}+|A|^{-2}}{2} x, x\right\rangle^{1 / 2}\left\langle\frac{|A|^{2}+|A|^{-2}}{2} y, y\right\rangle^{1 / 2} \\
& \geq\|x\|\|y\|+\left|\left\langle\frac{|A|^{2}+|A|^{-2}}{2} x, y\right\rangle-\langle x, y\rangle\right| \\
& \left\langle\frac{|A|^{2}+|A|^{-2}}{2} x, x\right\rangle^{1 / 2}\left\langle\frac{|A|^{2}+|A|^{-2}}{2} y, y\right\rangle^{1 / 2} \\
& -\left|\left\langle\frac{|A|^{2}+|A|^{-2}}{2} x, y\right\rangle\right| \geq\|x\|\|y\|-|\langle x, y\rangle| \geq 0 \\
& \left\langle\frac{|A|^{2}+|A|^{-2}}{2} x, x\right\rangle^{1 / 2}\left\langle\frac{|A|^{2}+|A|^{-2}}{2} y, y\right\rangle^{1 / 2} \\
& +\left|\left\langle\frac{|A|^{2}+|A|^{-2}}{2} x, y\right\rangle\right| \geq\|x\|\|y\|+|\langle x, y\rangle|
\end{aligned}
$$


and

$$
\begin{aligned}
& \frac{1}{2}\left[\left\langle\frac{|A|^{2}+|A|^{-2}}{2} x, x\right\rangle^{1 / 2}\left\langle\frac{|A|^{2}+|A|^{-2}}{2} y, y\right\rangle^{1 / 2}\right. \\
& \left.+\left|\left\langle\frac{|A|^{2}+|A|^{-2}}{2} x, y\right\rangle\right|\right] \geq|\langle x, y\rangle|
\end{aligned}
$$

for any $x, y \in H$.

If $A, B \geq 0$ with $A B=B A$, then from (10) we have

$$
\begin{aligned}
& \left\langle\frac{A^{2}+B^{2}}{2} x, x\right\rangle^{1 / 2}\left\langle\frac{A^{2}+B^{2}}{2} y, y\right\rangle^{1 / 2} \\
& \geq\langle A B x, x\rangle^{1 / 2}\langle A B y, y\rangle^{1 / 2}+\left|\left\langle\frac{A^{2}+B^{2}}{2} x, y\right\rangle-\langle A B x, y\rangle\right|, \\
& \left\langle\frac{A^{2}+B^{2}}{2} x, x\right\rangle^{1 / 2}\left\langle\frac{A^{2}+B^{2}}{2} y, y\right\rangle^{1 / 2}-\left|\left\langle\frac{A^{2}+B^{2}}{2} x, y\right\rangle\right| \\
& \geq\langle A B x, x\rangle^{1 / 2}\langle A B y, y\rangle^{1 / 2}-|\langle A B x, y\rangle| \geq 0, \\
& \left\langle\frac{A^{2}+B^{2}}{2} x, x\right\rangle^{1 / 2}\left\langle\frac{A^{2}+B^{2}}{2} y, y\right\rangle^{1 / 2}+\left|\left\langle\frac{A^{2}+B^{2}}{2} x, y\right\rangle\right| \\
& \geq\langle A B x, x\rangle^{1 / 2}\langle A B y, y\rangle^{1 / 2}+|\langle A B x, y\rangle|
\end{aligned}
$$

and

$$
\begin{aligned}
& \frac{1}{2}\left[\left\langle\frac{A^{2}+B^{2}}{2} x, x\right\rangle^{1 / 2}\left\langle\frac{A^{2}+B^{2}}{2} y, y\right\rangle^{1 / 2}\right. \\
& \left.+\left|\left\langle\frac{A^{2}+B^{2}}{2} x, y\right\rangle\right|\right] \geq|\langle A B x, y\rangle|
\end{aligned}
$$

for any $x, y \in H$.

We observe that if $A=1_{H}$ and $B=P$, with $P$ a projection on $H$, then we obtain from (32)

$$
\frac{1}{2}\left[\left\langle\frac{1_{H}+P}{2} x, x\right\rangle^{1 / 2}\left\langle\frac{1_{H}+P}{2} y, y\right\rangle^{1 / 2}+\left|\left\langle\frac{1_{H}+P}{2} x, y\right\rangle\right|\right] \geq|\langle P x, y\rangle|
$$

for any $x, y \in \mathrm{H}$.

If $e \in \mathrm{H},\|e\|=1$ then by taking $\mathrm{P}=\mathrm{P}_{e}$ defined in the introduction, we get the inequality

$$
\begin{aligned}
& \frac{1}{4}\left[\left[\|x\|^{2}+|\langle x, e\rangle|^{2}\right]^{1 / 2}\left[\|y\|^{2}+|\langle y, e\rangle|^{2}\right]^{1 / 2}+|\langle x, y\rangle+\langle x, e\rangle\langle e, y\rangle|\right] \\
& \quad \geq|\langle x, e\rangle\langle e, y\rangle|
\end{aligned}
$$


for any $x, y \in \mathrm{H}$.

Since

$$
|\langle x, y\rangle+\langle x, e\rangle\langle e, y\rangle| \leq|\langle x, y\rangle|+|\langle x, e\rangle\langle e, y\rangle|
$$

then by (34) we have

$\frac{1}{4}\left[\left[\|x\|^{2}+|\langle x, e\rangle|^{2}\right]^{1 / 2}\left[\|y\|^{2}+|\langle y, e\rangle|^{2}\right]^{1 / 2}+|\langle x, y\rangle|+|\langle x, e\rangle\langle e, y\rangle|\right] \geq|\langle x, e\rangle\langle e, y\rangle|$, which implies that

$$
\frac{1}{3}\left(\left[\|x\|^{2}+|\langle x, e\rangle|^{2}\right]^{1 / 2}\left[\|y\|^{2}+|\langle y, e\rangle|^{2}\right]^{1 / 2}+|\langle x, y\rangle|\right) \geq|\langle x, e\rangle\langle e, y\rangle|
$$

for any $x, y \in H$.

We recall that $\mathrm{U}: \mathrm{H} \rightarrow \mathrm{H}$ is a unitary operator if $\mathrm{U}^{*} \mathrm{U}=\mathrm{UU}^{*}=1_{\mathrm{H}}$. If $\mathrm{U}$ and $\mathrm{V}$ are unitary operators with $\operatorname{Re}\left(\mathrm{V}^{*} \mathrm{U}\right) \geq 0$, then by (20) we have

$$
\frac{1}{2}[\|x\|\|y\|+|\langle x, y\rangle|] \geq\left|\left\langle\operatorname{Re}\left(V^{*} u\right) x, y\right\rangle\right|
$$

for any $x, y \in H$.

In particular, if $\mathrm{U}$ is a unitary operator with $\operatorname{Re}(\mathrm{U}) \geq 0$ then by taking $\mathrm{V}=1_{\mathrm{H}}$ in $(36)$ we get

$$
\frac{1}{2}[\|x\|\|y\|+|\langle x, y\rangle|] \geq|\langle\operatorname{Re}(\mathrm{U}) x, y\rangle|
$$

for any $x, y \in H$.

\section{Inequalities for norm and numerical radius}

Let $(\mathrm{H} ;\langle\cdot, \cdot\rangle)$ be a complex Hilbert space. The numerical range of an operator $\mathrm{T}$ is the subset of the complex numbers $\mathbb{C}$ given by $[27$, p. 1$]$ :

$$
W(T)=\{\langle T x, x\rangle, x \in H,\|x\|=1\} .
$$

The numerical radius $w(\mathrm{~T})$ of an operator $\mathrm{T}$ on $\mathrm{H}$ is defined by $[27, \mathrm{p} .8]$ :

$$
w(T)=\sup \{|\lambda|, \lambda \in W(T)\}=\sup \{|\langle T x, x\rangle|,\|x\|=1\} .
$$

It is well known that $w(\cdot)$ is a norm on the Banach algebra $\mathrm{B}(\mathrm{H})$ and the following inequality holds true

$$
w(\mathrm{~T}) \leq\|\mathrm{T}\| \leq 2 w(\mathrm{~T}), \text { for any } \mathrm{T} \in \mathrm{B}(\mathrm{H}) .
$$

Utilising Buzano's inequality (3) we obtained the following inequality for the numerical radius [13] or [14]: 
Theorem 2 Let $(\mathrm{H} ;\langle\cdot, \cdot\rangle)$ be a Hilbert space and $\mathrm{T}: \mathrm{H} \rightarrow \mathrm{H}$ a bounded linear operator on $\mathrm{H}$. Then

$$
w^{2}(\mathrm{~T}) \leq \frac{1}{2}\left[w\left(\mathrm{~T}^{2}+\|\mathrm{T}\|^{2}\right] .\right.
$$

The constant $\frac{1}{2}$ is best possible in (38).

The following general result for the product of two operators holds [27, p. 37]:

Theorem 3 If $\mathrm{U}, \mathrm{V}$ are two bounded linear operators on the Hilbert space $(\mathrm{H},\langle\cdot, \cdot\rangle)$, then $w(\mathrm{UV}) \leq 4 w(\mathrm{U}) w(\mathrm{~V})$. In the case that $\mathrm{UV}=\mathrm{VU}$, then $w(\mathrm{UV}) \leq 2 w(\mathrm{U}) w(\mathrm{~V})$. The constant 2 is best possible here.

The following results are also well known [27, p. 38].

Theorem 4 If $\mathrm{U}$ is a unitary operator that commutes with another operator $\mathrm{V}$, then

$$
w(\mathrm{UV}) \leq w(\mathrm{~V}) .
$$

If $\mathrm{U}$ is an isometry and $\mathrm{UV}=\mathrm{VU}$, then (39) also holds true.

We say that $\mathrm{U}$ and $\mathrm{V}$ double commute if $\mathrm{UV}=\mathrm{VU}$ and $\mathrm{UV}^{*}=\mathrm{V}^{*} \mathrm{U}$. The following result holds $[27$, p. 38].

Theorem 5 If the operators $\mathrm{U}$ and $\mathrm{V}$ double commute, then

$$
w(\mathrm{UV}) \leq w(\mathrm{~V})\|\mathrm{U}\|
$$

As a consequence of the above, we have [27, p. 39]:

Corollary 2 Let $\mathrm{U}$ be a normal operator commuting with $\mathrm{V}$. Then

$$
w(\mathrm{UV}) \leq w(\mathrm{U}) w(\mathrm{~V}) .
$$

A related problem with the inequality (40) is to find the best constant $\mathrm{c}$ for which the inequality

$$
w(\mathrm{UV}) \leq \mathrm{cw}(\mathrm{U})\|\mathrm{V}\|
$$

holds for any two commuting operators $\mathrm{U}, \mathrm{V} \in \mathrm{B}(\mathrm{H})$. It is known that $1.064<$ $\mathrm{c}<1.169$, see [3], [35] and [36].

In relation to this problem, it has been shown in [25] that: 
Theorem 6 For any $\mathrm{U}, \mathrm{V} \in \mathrm{B}(\mathrm{H})$ we have

$$
w\left(\frac{\mathrm{UV}+\mathrm{VU}}{2}\right) \leq \sqrt{2} w(\mathrm{U})\|\mathrm{V}\| .
$$

For other numerical radius inequalities see the recent monograph [18] and the references therein.

Theorem 7 Let A, B two bounded linear operators on $\mathrm{H}$ such that $\operatorname{Re}\left(\mathrm{B}^{*} \mathrm{~A}\right)$ is a nonnegative operator. Then for any $\mathrm{U}, \mathrm{V} \in \mathrm{B}(\mathrm{H})$ we have

$$
\begin{aligned}
\left\|\operatorname{VRe}\left(\mathrm{B}^{*} \mathrm{~A}\right) \mathrm{U}\right\| \leq & \frac{1}{2}\left\|\left(\frac{|\mathrm{A}|^{2}+|\mathrm{B}|^{2}}{2}\right)^{1 / 2} \mathrm{u}\right\|\left\|\mathrm{v}\left(\frac{|\mathrm{A}|^{2}+|\mathrm{B}|^{2}}{2}\right)^{1 / 2}\right\| \\
& +\frac{1}{2}\left\|\mathrm{v}\left(\frac{|\mathrm{A}|^{2}+|\mathrm{B}|^{2}}{2}\right) \mathrm{u}\right\|, \\
w\left(\operatorname{VRe}\left(\mathrm{B}^{*} \mathrm{~A}\right) \mathrm{U}\right) \leq & \frac{1}{2}\left\|\left(\frac{|\mathrm{A}|^{2}+|\mathrm{B}|^{2}}{2}\right)^{1 / 2} \mathrm{u}\right\|\left\|\mathrm{v}\left(\frac{|\mathrm{A}|^{2}+|\mathrm{B}|^{2}}{2}\right)^{1 / 2}\right\| \\
& +\frac{1}{2} w\left(\mathrm{v}\left(\frac{|\mathrm{A}|^{2}+|\mathrm{B}|^{2}}{2}\right) \mathrm{u}\right)
\end{aligned}
$$

and

$$
\begin{aligned}
w\left(\operatorname{VRe}\left(\mathrm{B}^{*} \mathrm{~A}\right) \mathrm{U}\right) \leq & \frac{1}{4}\left\|\left.\left(\frac{|\mathrm{A}|^{2}+|\mathrm{B}|^{2}}{2}\right)^{1 / 2} \mathrm{u}\right|^{2}+\left|\left(\frac{|\mathrm{A}|^{2}+|\mathrm{B}|^{2}}{2}\right)^{1 / 2} \mathrm{~V}^{*}\right|^{2}\right\| \\
& +\frac{1}{2} w\left(\mathrm{v}\left(\frac{|\mathrm{A}|^{2}+|\mathrm{B}|^{2}}{2}\right) \mathrm{u}\right) .
\end{aligned}
$$

Proof. From the inequality (20) we have

$$
\begin{aligned}
\left|\left\langle\operatorname{Re}\left(B^{*} \mathrm{~A}\right) \mathrm{U} x, \mathrm{~V}^{*} \mathrm{y}\right\rangle\right| \leq & \frac{1}{2}\left[\left\langle\frac{|\mathrm{A}|^{2}+|\mathrm{B}|^{2}}{2} \mathrm{Ux}, \mathrm{Ux}\right\rangle^{1 / 2}\left\langle\frac{|\mathrm{A}|^{2}+|\mathrm{B}|^{2}}{2} \mathrm{~V}^{*} \mathrm{y}, \mathrm{V}^{*} \mathrm{y}\right\rangle^{1 / 2}\right. \\
& \left.+\left|\left\langle\frac{|\mathrm{A}|^{2}+|B|^{2}}{2} \mathrm{Ux}, \mathrm{V}^{*} \mathrm{y}\right\rangle\right|\right]
\end{aligned}
$$

for any $x, y \in H$, which is equivalent to

$$
\begin{aligned}
& \left|\left\langle\operatorname{VRe}\left(B^{*} A\right) U x, y\right\rangle\right| \\
& \leq \frac{1}{2}\left[\left\langle U^{*} \frac{|A|^{2}+|B|^{2}}{2} \mathrm{Ux}, x\right\rangle^{1 / 2}\left\langle\mathrm{~V} \frac{|A|^{2}+|B|^{2}}{2} V^{*} y, y\right\rangle^{1 / 2}\right. \\
& \left.\quad+\left|\left\langle V \frac{|A|^{2}+|B|^{2}}{2} \mathrm{U} x, y\right\rangle\right|\right]
\end{aligned}
$$


for any $x, y \in H$.

Taking the supremum over $x, y \in H,\|x\|=\|y\|=1$ we have

$$
\begin{aligned}
\| & \operatorname{Re}\left(\mathrm{B}^{*} \mathrm{~A}\right) \mathrm{U} \|=\sup _{\|x\|=\|y\|=1}\left|\left\langle\operatorname{VRe}\left(\mathrm{B}^{*} \mathrm{~A}\right) \mathrm{U} x, y\right\rangle\right| \\
\leq & \frac{1}{2} \sup _{\|x\|=\|y\|=1}\left[\left\langle\mathrm{U}^{*} \frac{|\mathrm{A}|^{2}+|\mathrm{B}|^{2}}{2} \mathrm{U} x, x\right\rangle^{1 / 2}\left\langle\mathrm{~V} \frac{|\mathrm{A}|^{2}+|\mathrm{B}|^{2}}{2} \mathrm{~V}^{*} \mathrm{y}, \mathrm{y}\right\rangle^{1 / 2}\right. \\
& \left.+\left|\left\langle\mathrm{V} \frac{|\mathrm{A}|^{2}+|\mathrm{B}|^{2}}{2} \mathrm{U} x, y\right\rangle\right|\right] \\
\leq & \frac{1}{2}\left[\sup _{\|x\|=1}\left\langle\mathrm{U}^{*} \frac{|\mathrm{A}|^{2}+|\mathrm{B}|^{2}}{2} \mathrm{U} x, x\right\rangle^{1 / 2} \sup _{\|y\|=1}\left\langle\mathrm{~V} \frac{|\mathrm{A}|^{2}+|\mathrm{B}|^{2}}{2} \mathrm{~V}^{*} \mathrm{y}, \mathrm{y}\right\rangle^{1 / 2}\right. \\
& \left.+\sup _{\|x\|=\|y\|=1}\left|\left\langle\mathrm{~V} \frac{|\mathrm{A}|^{2}+|\mathrm{B}|^{2}}{2} \mathrm{Ux}, \mathrm{y}\right\rangle\right|\right] \\
= & \frac{1}{2}\left[\left\|\mathrm{U}^{*} \frac{|\mathrm{A}|^{2}+|\mathrm{B}|^{2}}{2} \mathrm{U}\right\|^{1 / 2}\left\|\mathrm{~V} \frac{|\mathrm{A}|^{2}+|\mathrm{B}|^{2}}{2} \mathrm{~V}^{*}\right\|^{1 / 2}+\left\|\mathrm{V} \frac{|\mathrm{A}|^{2}+|\mathrm{B}|^{2}}{2} \mathrm{u}\right\|\right] .
\end{aligned}
$$

Since

$$
\mathrm{u}^{*} \frac{|\mathrm{A}|^{2}+|\mathrm{B}|^{2}}{2} \mathrm{U}=\left|\left(\frac{|\mathrm{A}|^{2}+|\mathrm{B}|^{2}}{2}\right)^{1 / 2} \mathrm{u}\right|^{2}
$$

and

$$
\mathrm{V} \frac{|\mathrm{A}|^{2}+|\mathrm{B}|^{2}}{2} \mathrm{~V}^{*}=\left|\left(\frac{|\mathrm{A}|^{2}+|\mathrm{B}|^{2}}{2}\right)^{1 / 2} \mathrm{~V}^{*}\right|^{2}
$$

then

$$
\left\|\mathrm{u}^{*} \frac{|\mathrm{A}|^{2}+|\mathrm{B}|^{2}}{2} \mathrm{u}\right\|^{1 / 2}=\left\|\left(\frac{|\mathrm{A}|^{2}+|\mathrm{B}|^{2}}{2}\right)^{1 / 2} \mathrm{u}\right\|
$$

and

$$
\left\|V \frac{|A|^{2}+|B|^{2}}{2} V^{*}\right\|^{1 / 2}=\left\|\left(\frac{|A|^{2}+|B|^{2}}{2}\right)^{1 / 2} V^{*}\right\|=\left\|V\left(\frac{|A|^{2}+|B|^{2}}{2}\right)^{1 / 2}\right\| .
$$

Using (46) we also have

$$
\begin{aligned}
\left|\left\langle\operatorname{VRe}\left(\mathrm{B}^{*} \mathrm{~A}\right) \mathrm{U} x, x\right\rangle\right| \leq & \frac{1}{2}\left[\left\langle\mathrm{U}^{*} \frac{|\mathrm{A}|^{2}+|\mathrm{B}|^{2}}{2} \mathrm{U} x, x\right\rangle^{1 / 2}\left\langle\mathrm{~V} \frac{|\mathrm{A}|^{2}+|\mathrm{B}|^{2}}{2} \mathrm{~V}^{*} x, x\right\rangle^{1 / 2}\right. \\
& \left.+\left|\left\langle\mathrm{V} \frac{|\mathrm{A}|^{2}+|\mathrm{B}|^{2}}{2} \mathrm{U} x, x\right\rangle\right|\right]
\end{aligned}
$$


for any $x \in H,\|x\|=1$.

Taking the supremum over $x \in H,\|x\|=1$ we have

$$
\begin{aligned}
& w\left(\operatorname{VRe}\left(\mathrm{B}^{*} \mathrm{~A}\right) \mathrm{U}\right)=\sup _{\|x\|=1}\left|\left\langle\operatorname{VRe}\left(\mathrm{B}^{*} \mathrm{~A}\right) \mathrm{U} x, x\right\rangle\right| \\
& \leq \frac{1}{2}\left[\sup _{\|x\|=1}\left\langle\mathrm{U}^{*} \frac{|\mathrm{A}|^{2}+|\mathrm{B}|^{2}}{2} \mathrm{U} x, x\right\rangle^{1 / 2} \sup _{\|x\|=1}\left\langle\mathrm{~V} \frac{|\mathrm{A}|^{2}+|\mathrm{B}|^{2}}{2} \mathrm{~V}^{*} x, x\right\rangle^{1 / 2}\right. \\
& \left.\quad+\sup _{\|x\|=1} \mid\left\langle\mathrm{V} \frac{|\mathrm{A}|^{2}+|\mathrm{B}|^{2}}{2} \mathrm{U} x, x\right\rangle\right] \\
& =\frac{1}{2}\left[\left\|\left(\frac{|\mathrm{A}|^{2}+|\mathrm{B}|^{2}}{2}\right)^{1 / 2} \mathrm{u}\right\|\left\|\mathrm{v}\left(\frac{|\mathrm{A}|^{2}+|\mathrm{B}|^{2}}{2}\right)^{1 / 2}\right\|+w\left(\mathrm{~V} \frac{|\mathrm{A}|^{2}+|\mathrm{B}|^{2}}{2} \mathrm{U}\right)\right]
\end{aligned}
$$

and the inequality (44) is proved.

By the arithmetic mean - geometric mean inequality we have

$$
\begin{aligned}
& \left\langle\mathrm{U}^{*} \frac{|\mathrm{A}|^{2}+|\mathrm{B}|^{2}}{2} \mathrm{U} x, x\right\rangle^{1 / 2}\left\langle\mathrm{~V} \frac{|\mathrm{A}|^{2}+|\mathrm{B}|^{2}}{2} \mathrm{~V}^{*} x, x\right\rangle^{1 / 2} \\
& \leq \frac{1}{2}\left[\left\langle\mathrm{U}^{*} \frac{|\mathrm{A}|^{2}+|\mathrm{B}|^{2}}{2} \mathrm{U} x, x\right\rangle+\left\langle\mathrm{V} \frac{|\mathrm{A}|^{2}+|\mathrm{B}|^{2}}{2} \mathrm{~V}^{*} x, x\right\rangle\right] \\
& =\frac{1}{2}\left\langle\left[\left|\left(\frac{|\mathrm{A}|^{2}+|\mathrm{B}|^{2}}{2}\right)^{1 / 2} \mathrm{u}\right|^{2}+\left|\left(\frac{|\mathrm{A}|^{2}+|\mathrm{B}|^{2}}{2}\right)^{1 / 2} \mathrm{~V}^{*}\right|^{2}\right] x, x\right\rangle
\end{aligned}
$$

for any $x \in H,\|x\|=1$.

From (48) we have

$$
\begin{aligned}
& \left|\left\langle\operatorname{VRe}\left(\mathrm{B}^{*} \mathrm{~A}\right) \mathrm{U} x, x\right\rangle\right| \\
& \leq \frac{1}{4}\left\langle\left[\left|\left(\frac{|\mathrm{A}|^{2}+|\mathrm{B}|^{2}}{2}\right)^{1 / 2} \mathrm{u}\right|^{2}+\left|\left(\frac{|\mathrm{A}|^{2}+|\mathrm{B}|^{2}}{2}\right)^{1 / 2} \mathrm{~V}^{*}\right|^{2}\right] x, x\right\rangle \\
& \quad+\frac{1}{2}\left|\left\langle\mathrm{~V} \frac{|\mathrm{A}|^{2}+|\mathrm{B}|^{2}}{2} \mathrm{U} x, x\right\rangle\right|
\end{aligned}
$$

for any $x \in H,\|x\|=1$.

Taking the supremum over $x \in H,\|x\|=1$ in (51) we get the desired inequality (45). 
Corollary 3 If $\mathrm{A}, \mathrm{B} \geq 0$ with $\mathrm{AB}=\mathrm{BA}$, then for any $\mathrm{U}, \mathrm{V} \in \mathrm{B}(\mathrm{H})$ we have

$$
\begin{aligned}
\| \text { VABU } \| \leq & \frac{1}{2}\left\|\left(\frac{A^{2}+B^{2}}{2}\right)^{1 / 2} \mathrm{u}\right\|\left\|v\left(\frac{A^{2}+B^{2}}{2}\right)^{1 / 2}\right\| \\
& +\frac{1}{2}\left\|v\left(\frac{A^{2}+B^{2}}{2}\right) u\right\|, \\
w(\text { VABU }) \leq & \frac{1}{2}\left\|\left(\frac{A^{2}+B^{2}}{2}\right)^{1 / 2} u\right\|\left\|v\left(\frac{A^{2}+B^{2}}{2}\right)^{1 / 2}\right\| \\
& +\frac{1}{2} w\left(v\left(\frac{A^{2}+B^{2}}{2}\right) u\right)
\end{aligned}
$$

and

$$
\begin{aligned}
w(\text { VABU }) \leq & \frac{1}{4}\left\|\left|\left(\frac{A^{2}+B^{2}}{2}\right)^{1 / 2} \mathrm{u}\right|^{2}+\left|\left(\frac{A^{2}+B^{2}}{2}\right)^{1 / 2} \mathrm{~V}^{*}\right|^{2}\right\| \\
& +\frac{1}{2} w\left(v\left(\frac{A^{2}+B^{2}}{2}\right) \mathrm{u}\right) .
\end{aligned}
$$

Remark 2 If we take in Corollary $3 \mathrm{~A}=1_{\mathrm{H}}$ and $\mathrm{B}=\mathrm{P}$, a projection on $\mathrm{H}$, then we get

$$
\begin{aligned}
\|\mathrm{VPU}\| \leq & \frac{1}{2}\left\|\left(\frac{1_{\mathrm{H}}+\mathrm{P}}{2}\right)^{1 / 2} \mathrm{u}\right\|\left\|\mathrm{V}\left(\frac{1_{\mathrm{H}}+\mathrm{P}}{2}\right)^{1 / 2}\right\| \\
& +\frac{1}{2}\left\|\mathrm{~V}\left(\frac{1_{\mathrm{H}}+\mathrm{P}}{2}\right) \mathrm{u}\right\|, \\
w(\mathrm{VPU}) \leq & \frac{1}{2}\left\|\left(\frac{1_{\mathrm{H}}+\mathrm{P}}{2}\right)^{1 / 2} \mathrm{u}\right\|\left\|\mathrm{v}\left(\frac{1_{\mathrm{H}}+\mathrm{P}}{2}\right)^{1 / 2}\right\| \\
& +\frac{1}{2} w\left(\mathrm{~V}\left(\frac{1_{\mathrm{H}}+\mathrm{P}}{2}\right) \mathrm{u}\right)
\end{aligned}
$$

and

$$
\begin{aligned}
w \mathrm{VPU}) \leq & \frac{1}{4}\left\|\left|\left(\frac{1_{\mathrm{H}}+\mathrm{P}}{2}\right)^{1 / 2} \mathrm{u}\right|^{2}+\left|\left(\frac{1_{\mathrm{H}}+\mathrm{P}}{2}\right)^{1 / 2} \mathrm{~V}^{*}\right|^{2}\right\| \\
& +\frac{1}{2} w\left(\mathrm{~V}\left(\frac{1_{\mathrm{H}}+\mathrm{P}}{2}\right) \mathrm{U}\right) .
\end{aligned}
$$

Finally, we have: 
Corollary 4 Let $\mathrm{T}$ be a unitary operator with $\operatorname{Re}(\mathrm{T}) \geq 0$. Then for any $\mathrm{U}, \mathrm{V} \in$ $\mathrm{B}(\mathrm{H})$ we have

$$
\begin{gathered}
\|\operatorname{VRe}(\mathrm{T}) \mathrm{U}\| \leq \frac{1}{2}[\|\mathrm{U}\|\|\mathrm{V}\|+\|\mathrm{Vu}\|], \\
w(\operatorname{VRe}(\mathrm{T}) \mathrm{U}) \leq \frac{1}{2}[\|\mathrm{U}\|\|\mathrm{V}\|+w(\mathrm{Vu})]
\end{gathered}
$$

and

$$
w(\operatorname{VRe}(\mathrm{T}) \mathrm{U}) \leq \frac{1}{4}\left\||\mathrm{U}|^{2}+\left|\mathrm{V}^{*}\right|^{2}\right\|+\frac{1}{2} w(\mathrm{VU})
$$

\section{References}

[1] J. M. Aldaz, Strengthened Cauchy-Schwarz and Hölder inequalities. J. Inequal. Pure Appl. Math. 10 (4) (2009), Article 116, 6 pp.

[2] M. L. Buzano, Generalizzazione della diseguaglianza di Cauchy-Schwarz. (Italian), Rend. Sem. Mat. Univ. e Politech. Torino, 31 (1971/73) (1974), 405-409.

[3] K. R. Davidson, J. A. R. Holbrook, Numerical radii of zero-one matricies, Michigan Math. J. 35 (1988), 261-267.

[4] A. De Rossi, A strengthened Cauchy-Schwarz inequality for biorthogonal wavelets. Math. Inequal. Appl., 2 (2) (1999), 263-282.

[5] S. S. Dragomir, Some refinements of Schwartz inequality, Simpozionul de Matematici şi Aplicaţii, Timişoara, Romania, 1-2 Noiembrie 1985, 13-16.

[6] S. S. Dragomir, A generalization of Grüss' inequality in inner product spaces and applications, J. Math. Anal. Appl., 237 (1999), 74-82.

[7] S. S. Dragomir, Some Grüss type inequalities in inner product spaces, $J$. Inequal. Pure and Appl. Math., 4 (2) Art. 42 (2003).

[8] S. S. Dragomir, Some inequalities for power series of selfadjoint operators in Hilbert spaces via reverses of the Schwarz inequality. Integral Transforms Spec. Funct. 20 (9-10) (2009), 757-767.

[9] S. S. Dragomir, A potpourri of Schwarz related inequalities in inner product spaces. I. J. Inequal. Pure Appl. Math. 6 (3) (2005), Article 59, 15 pp. 
[10] S. S. Dragomir, A potpourri of Schwarz related inequalities in inner product spaces. II. J. Inequal. Pure Appl. Math. 7 (1) (2006), Article 14, 11 pp.

[11] S. S. Dragomir, Reverses of Schwarz, triangle and Bessel inequalities in inner product spaces. J. Inequal. Pure Appl. Math. 5 (3) (2004), Article 76, 18 pp.

[12] S. S. Dragomir, New reverses of Schwarz, triangle and Bessel inequalities in inner product spaces. Aust. J. Math. Anal. Appl. 1 (1) (2004), Art. 1, $18 \mathrm{pp}$.

[13] S. S. Dragomir, Inequalities for the norm and the numerical radius of linear operators in Hilbert spaces. Demonstratio Math. 40 (2) (2007), $411-417$.

[14] S. S. Dragomir, Some inequalities for the norm and the numerical radius of linear operators in Hilbert spaces. Tamkang J. Math. 39 (1) (2008), $1-7$.

[15] S. S. Dragomir, New inequalities of the Kantorovich type for bounded linear operators in Hilbert spaces, Linear Algebra and its Applications 428 (2008), 2750-2760.

[16] S. S. Dragomir, Advances in Inequalities of the Schwarz, Grüss and Bessel Type in Inner Product Spaces. Nova Science Publishers, Inc., Hauppauge, NY, 2005. viii+249 pp. ISBN: 1-59454-202-3.

[17] S. S. Dragomir, Advances in Inequalities of the Schwarz, Triangle and Heisenberg Type in Inner Product Spaces. Nova Science Publishers, Inc.,

[18] S. S. Dragomir, Inequalities for the Numerical Radius of Linear Operators in Hilbert Spaces. Springer Briefs in Mathematics. Springer, 2013. $\mathrm{x}+120$ pp. ISBN: 978-3-319-01447-0; 978-3-319-01448-7.

[19] S. S. Dragomir, Some inequalities in inner product spaces related to Buzano's and Grüss' results, Preprint, RGMIA Res. Rep. Coll., 18 (2015), Art. 16. [Online http://rgmia.org/papers/v18/v18a16.pdf] .

[20] S. S. Dragomir, Some inequalities in inner product spaces, Preprint, RGMIA Res. Rep. Coll., 18 (2015), Art. 19. [Online http://rgmia.org/papers/v18/v18a19.pdf] 
[21] S. S. Dragomir, Vector inequalities for a projection in Hilbert spaces and applications, Preprint, RGMIA Res. Rep. Coll., 18 (2015), Art.

[22] S. S. Dragomir, Anca C. Goşa, Quasilinearity of some composite functionals associated to Schwarz's inequality for inner products. Period. Math. Hungar. 64 (1) (2012), 11-24.

[23] S. S. Dragomir, B. Mond, Some mappings associated with CauchyBuniakowski-Schwarz's inequality in inner product spaces. Soochow J. Math. 21 (4) (1995), 413-426.

[24] S. S. Dragomir, I. Sándor, Some inequalities in pre-Hilbertian spaces. Studia Univ. Babę-Bolyai Math. 32 (1) (1987), 71-78.

[25] C. K. Fong, J. A. R. Holbrook, Unitarily invariant operators norms, Canad. J. Math. 35 (1983), 274-299.

[26] H. Gunawan, On n-inner products, n-norms, and the Cauchy-Schwarz inequality. Sci. Math. Jpn. 55 (1) (2002), 53-60

[27] K. E. Gustafson, D. K. M. Rao, Numerical Range, Springer-Verlag, New York, Inc., 1997.

[28] P. R. Halmos, A Hilbert Space Problem Book, Springer-Verlag, New York, Heidelberg, Berlin, Second edition, 1982.

[29] J. A. R. Holbrook, Multiplicative properties of the numerical radius in operator theory, J. Reine Angew. Math. 237 (1969), 166-174.

[30] E. R. Lorch, The Cauchy-Schwarz inequality and self-adjoint spaces. Ann. of Math. (2) 46, (1945), 468-473.

[31] C. Lupu, D. Schwarz, Another look at some new Cauchy-Schwarz type inner product inequalities. Appl. Math. Comput. 231 (2014), 463-477.

[32] M. Marcus, The Cauchy-Schwarz inequality in the exterior algebra. Quart. J. Math. Oxford Ser. (2) 17 (1966), 61-63.

[33] P. R. Mercer, A refined Cauchy-Schwarz inequality. Internat. J. Math. Ed. Sci. Tech. 38 (6) (2007), 839-842.

[34] F. T. Metcalf, A Bessel-Schwarz inequality for Gramians and related bounds for determinants. Ann. Mat. Pura Appl. (4) 68 (1965), 201-232. 
[35] V. Müller, The numerical radius of a commuting product, Michigan Math. J. 39 (1988), 255-260.

[36] K. Okubo, T. Ando, Operator radii of commuting products, Proc. Amer. Math. Soc. 56 (1976), 203-210.

[37] T. Precupanu, On a generalization of Cauchy-Buniakowski-Schwarz inequality. An. Şti. Univ. "Al. I. Cuza" Iaşi Secţ. I a Mat. (N.S.) 22 (2) (1976), 173-175.

[38] K. Trenčevski, R. Malčeski, On a generalized n-inner product and the corresponding Cauchy-Schwarz inequality. J. Inequal. Pure Appl. Math. 7 (2) (2006), Article 53, 10 pp.

[39] G.-B. Wang, J.-P. Ma, Some results on reverses of Cauchy-Schwarz inequality in inner product spaces. Northeast. Math. J. 21 (2) (2005), 207211. 